\title{
Long-term recognition memory for auditory patterns*
}

\author{
IRWIN M. SPIGEL $\div$. MALCOLM NOVAR \\ and BRENDA NOVAR \\ Erindale College. Lniversity of Toronto \\ Toronto. Ont. Canada
}

The superiority of recognition memory for 5-tone over 3-tone auditory patterns after a 24-h interval was shown, regardless of whether the distractor sequence in the test session was the reverse of the standard or an identical pattern four half-tones higher in frequency. Twenty presentations of the standard in the initial session yielded better recognition than a single presentation, but both were superior to a single presentation with Ss asked to "hum" the pattern aloud immediately after listening to it. The problem of attributing the results merely to increased structure is raised, as is the possible role of interference in the memory decrement observed in the "hum" condition.

While tonal pitch has received its share of attention in the broad effort to examine memory for nonverbal material. retention of patterned auditory stimulation has attracted relatively little concentration. The work of Royer \& Garner (1966.1970) focused on the perceptual organization of auditory temporal patterns. but utilized only two stimulus frequencies. Wohlwill (1971) observed that young children evidenced great difficulty in learning auditory patterns. and only at later stages of development could make effective use of correlated information in other sense modalities.

Interference effects in the immediate memory for pitch were demonstrated by Deutsch (1970). who concluded that such disruption was highly specific in nature and not traceable to limitations in short-term memory capacity or distraction. Deutsch (1972) later observed that intervening tones impaired recognition of test tones when the frequencies of these differed up to 2/3 tone. King (1963). on the other hand. found that tonal comparisons by Ss with a standard stimulus remained highly accurate with delays of $2 \mathrm{~min}$ to 28 days.

The purpose of the current study. though preliminary in nature. was to explore relatively long-term memory for more complex auditory patterns. Specifically. the recognition of 3-tone and 5-tone sequences after a $24-\mathrm{h}$ interval was examined under three conditions of initial stimulus presentation.

\section{METHOD}

\section{Subjects}

One hundred and twenty-four male and female students in the Liniversity Extension Division. ranging in age from 17 to 58 years. were employed on a voluntary basis. Assignment to @roups

\footnotetext{
*This research was internally funded br a speical grant from Erindale College. Univeristy of Toronto.

-Reprints may be obtained by writing to Inwin Spigel. Erindale College. University of Toronto. Toronto. Ont.. Canada.
}

representing 24 possible combinations of initial and test stimulus conditions was random. subject to the constraints imposed by maintaining near-equality in group size and sex distribution from the volunteer pool available.

\section{Apparatus}

For the purpose of simplicity. only two tonal sequences were employed-both selected from tones of the equal tempered scale (see Deutsch. 1972)-one each of lengths 3 and 5. Tones were produced by an RCA WA-44C audio generator and tape-recorded on a Sony TC 252 . Tones were all $250 \mathrm{msec}$ in duration. with 200 -msec intertonal intervals. In the one condition requiring multiple presentation. $1,500 \mathrm{msec}$ separated each delivery of the tonal pattern.

\section{Procedure}

Instructions and auditory stimulus patterns for initial and test sessions were taped and delivered to Ss by way of headsets from cassette units. In all cases, Ss appeared at the laboratory for two sessions, $24 \mathrm{~h}$ apart. None was informed of the real nature of the experiment, only that it was part of a study of auditory perception. Although there were 12 presentation-test situations studied. counterbalancing for order of probe and distractor sequences in the second (recognition) session required constitution of 24 groups. All Ss were assigned to one of six initial auditory pattern sequence conditions: As instructed. they listened to a single presentation of either the 3-tone sequence or the 5-tone sequence: 20 presentations, each separated by $1.500 \mathrm{msec}$. of the 3 -tone or 5 -tone pattern. or a single presentation of the 3-tone or 5-tone sequence, after which. as instructed. they were to "hum" the pattern they had just heard. Second-jession assionments of all $\mathrm{Ss}$ in both the 3-tone and 5-tone groups were to listen to two sequences of the same length and report whether the first or the second was the particular tonal pattern they had been presented $24 \mathrm{~h}$ earlier. In half the cases. the correct sequence was paired with one identical in tonal interval but four ${ }^{1}$ z-steps higher in the equal-tempered scale. In the remaining cases. the correct sequence was paired with a temporal pattern in reverse order to the original. To counterbalance for order. the correct pattern was presented first in half the cases, and the distractor preceding in the remaining half. The initial 3-tone sequence consisted of tones of 308.411 , and $290 \mathrm{~Hz}$. the four half-step removed distrastor sequence for which consisted of tones of 388,517 , and $366 \mathrm{~Hz}$. The 5-tone standard sequence consisted of tones of 366.290 .345 .411 . and $308 \mathrm{~Hz}$. the four half-step variant being 461.366 .435 .517 . and $388 \mathrm{~Hz}$. Preliminary sampling from independent members of the university community indicated that no specific associative value was attached to either of the sequences or their up-shifted or reversed variants.

\section{RESULTS AND DISCUSSION}

Binomial tests of "correct" vs "incorrect" recognitions were employed across all major dimensions of comparison. Table 1 includes the data for these dichotomous treatments. Clearly significant superiority. of recognition was observed when the distractor was the reverse of the standard pattern rather than merely the identical pattern raised four half-steps. Tables 1C. $1 \mathrm{D}$. $1 \mathrm{~F}$. and $1 \mathrm{G}$. however. reveal that this superiority was virtually entirely attributable to the performance of $\mathrm{Ss}$ with the s-tone sequences. none of the s-tone comparisons yielding anywhere near the recognition 
Table 1

Numbers of Correct and Incorrect Recognitions of Three- and Five-Tone Auditory Patterns under Three Conditions of Stimubation and with Distractors of Reversed or Raised Sequences

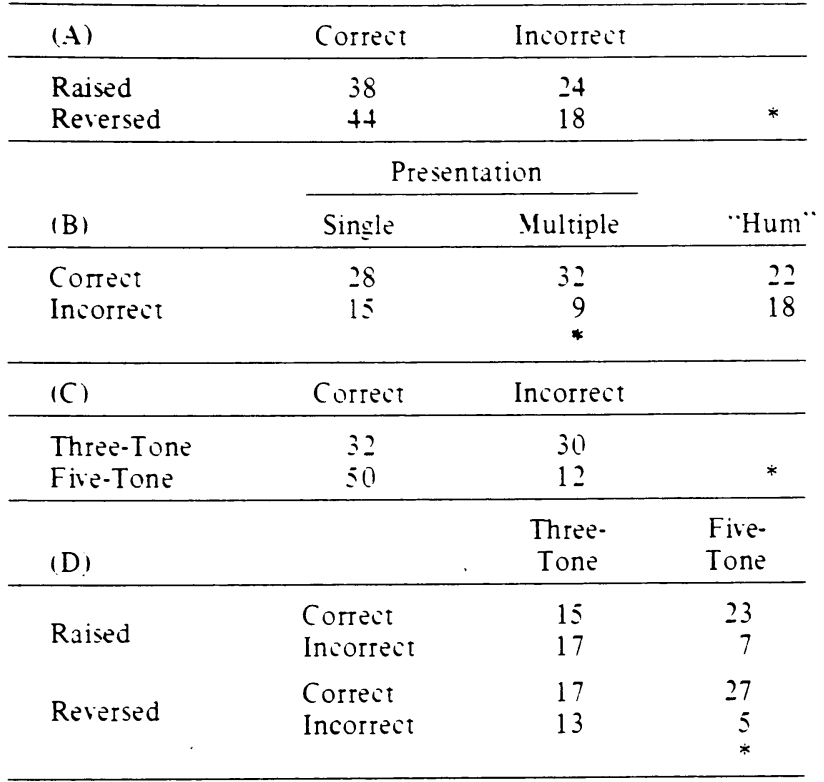

\begin{tabular}{llccc}
\hline & & \multicolumn{2}{c}{ Presentation } & \\
\cline { 3 - 4 }$($ E) & & Single & Multiple & "Hum \\
\hline \multirow{2}{*}{ Raised } & Correct & 12 & 17 & 9 \\
& Incorrect & 9 & 4 & 11 \\
\multirow{2}{*}{ Reversed } & Correct & 16 & 15 & 13 \\
& Incorrect & 6 & 5 & 7 \\
& & & $*$ & \\
\hline \multirow{2}{*}{ (F) } & & Single & Multiple & "Hum" \\
\hline
\end{tabular}

\begin{tabular}{clccc}
\hline Three-Tone Only & & & \\
Raised & Correct & 4 & 8 & 3 \\
& Incorrect & 7 & 3 & 7 \\
\multirow{2}{*}{ Reversed } & Correct & 6 & 6 & 5 \\
& Incorrect & 4 & 4 & 5 \\
\hline \multirow{2}{*}{$(\mathrm{G})$} & & Single & Multiple "Hum" \\
\hline
\end{tabular}

Five-Tone Only

\begin{tabular}{llrrr}
\multirow{2}{*}{ Raised } & Correct & 8 & 9 & 6 \\
\multirow{2}{*}{ Reversed } & Incorrect & 2 & 1 & 4 \\
& Correct & 10 & 9 & 8 \\
& Incorrect & 2 & 1 & 2
\end{tabular}

*Statistically significant

accuracy of the longer patterns. Table 1C. in fact. emphasizes the general improvement in recognition of 5 -tone over 3-tone auditory patterns. Tables $1 \mathrm{~B}$ and $1 \mathrm{E}$ further indicate that multiple presentation considerably improves long-term recognition of the auditory sequences. but it should be added that the data for single presentations (Table 1B) just failed to attain statistical significance. The condition requiring Ss to "hum" the pattern immediately after a single presentation produced purely chance recognition $24 \mathrm{~h}$ later.

The data reveal quite clearly the superiority of long-term recognition memory for 5-tone over 3-tone auditory patterns. such superiority evident when the distracting sequence is either the reverse of the standard or an identical pattern in tones four half-steps higher in frequency. Further analysis of the data shows. in fact. that virtually all differences observed in the experiment were attributable to the 5-tone data. Twenty presentations of the standard at the first session proves superior to a single presentation. but both are clearly superior to the situation where Ss are instructed to "hum" the pattern aloud after a single presentaiton of the standard.

It is tempting to infer from the data that the improved recognition memory for the 5-tone temporal pattern mav be a function of the increased structure of the longer standard. Such an explanation would appear to be valid with reference to standards with distractors of the sequence in reverse. It would seem likely. however. for the increased structure of longer patterns to facilitate recognition of sequences where only a four-half-step raise in frequency differentiated distractor from standard. as is evidenced in Tables $1 \mathrm{D}$ and $1 \mathrm{G}$. Nevertheless. the possibility that increased structure reduces whatever confusion results from small pitch shifts requires additional study. Should such prove to be the case. it would suggest the operation of processes other than those involved in the short-term recognition memory for familiar melodic patterns. Dowling \& Fujitani (1971) found that melodic contour became an important variable in recognition of standards against transposed comparisons, confusion being greatest when the latter were exact transpositions of the former. In short, structure did not appear to be an aid when pitch shift alone differentiated test patterns.

The superiority of a multiply presented standard is consistent with the data for memory of other forms of material. but the total failure of the "hum" condition seems to require some examination. It is possible that $\mathrm{Ss}$ generally are not able to reproduce tonal patterns with any great accuracy, and actually hummed an incorrect sequence. The processing of such incorrect information could conceivably have interfered with accurate recognition of the standard during the test session $24 \mathrm{~h}$ later-an effect somewhat analogous to that noted by Crowder (1970) with verbal material.

\section{REFERENCES}

Crowder, R: G. Role of one's own voice in immediate memory. Cognitive Psychology, 1970, 1, 157-158.

Deutsch. D. Tones and numbers: Specificity of interference in immediate memory. Science. 1970, 168, 1604-1605.

Deutsch. D. Mapping of interactions in the pitch memory store. Science, 1972.175. 1020-1022.

Dowling, W. J.. \& Fujitani. D. S. Contour. interial and pitch recognition in memory for melodies. Journal of the Acoustical Society of America, 1971, 49, 524-531.

King. H. E. The retention of sensory experience: II. Frequency. Journal of Psychology, 1963. 56, 291-298.

Rover, F. L.. \& Garner. W. R. Response uncretainty and perceptual difficulty of auditory temporal patterns. Perception \& Psychophysics. 1966, 1. 41-47.

Royer. F. L.. \& Garner. W. R. Perceptual organization of nine-element auditory temporal patterns. Perception \& Psychophysics. 1970. 7. 115-120.

Wohlwill. J. F. Effect of correlated visual and tactual feedback on auditory pattern learning at different age levels. Journal of Experimental Child Psychology. 1971. 11.213-228.

(Received for publication August 17. 1973.) 